\title{
A perspectiva dos pacientes internados na Unidade de Terapia Intensiva acerca da
}

\section{assistência fisioterapêutica}

\author{
The perspective of Intensive Care Unit patients about physical therapy care \\ La perspectiva de los pacientes hospitalizados en la Unidad de Cuidados Intensivos sobre los \\ cuidados fisioterapéuticos
}

Recebido: 05/07/2021 | Revisado: 10/07/2021 | Aceito: 14/07/2021 | Publicado: 24/07/2021

\begin{abstract}
Alyssa Ellen Peixoto Vidal
ORCID: https://orcid.org/0000-0002-6995-0587

Universidade Estadual do Sudoeste da Bahia, Brasi

E-mail: alyssa.ellen99@gmail.com

Karine Orrico Góes

ORCID: https://orcid.org/0000-0001-5011-2148

Universidade Estadual do Sudoeste da Bahia, Brasil

E-mail: karineorrico@yahoo.com.br

Giovanna Porto dos Santos

ORCID: https://orcid.org/0000-0002-6203-7437

Universidade Estadual do Sudoeste da Bahia, Brasil

E-mail: giovannaportofm@gmail.com

Priscila D'Almeida Ferreira

ORCID: https://orcid.org/0000-0001-8205-4691 Universidade Estadual do Sudoeste da Bahia, Brasil

E-mail: priuesb@yahoo.com.br

Nayara Alves de Sousa

ORCID: https://orcid.org/0000-0003-4746-0103 Universidade Estadual do Sudoeste da Bahia, Brasil

E-mail: nayara.sousa1@hotmail.com

Jamine Barros Oliveira Araújo

ORCID: https://orcid.org/0000-0003-1301-5597 Universidade Estadual do Sudoeste da Bahia, Brasil E-mail: jamine.barros@uesb.edu.br
\end{abstract}

\begin{abstract}
Resumo
A importância da assistência fisioterapêutica humanizada na Unidade de Terapia Intensiva é amplamente conhecida, bem como as percepções dos profissionais e familiares acerca da humanização. Entretanto, a perspectiva do paciente, que realmente está sendo assistido, ainda é pouco conhecida. A partir disso, esse estudo objetiva descrever a perspectiva dos pacientes internados acerca da assistência fisioterapêutica na Unidade de Terapia Intensiva, considerando aspectos da humanização. Trata-se de um estudo de corte transversal, descritivo, com abordagem quantitativa. Foi utilizado um questionário constituído por dois blocos de questões fechadas, sendo o primeiro para o conhecimento de dados sociodemográficos e o segundo para conhecimento da perspectiva do paciente acerca da assistência fisioterapêutica. A população foi composta por 20 pacientes adultos, com idade igual ou superior a 18 anos, internados na UTI por no mínimo 24 horas, lúcidos e orientados, e assistidos pela fisioterapia. Foi realizada uma análise descritiva simples e os resultados foram apresentados em forma de números absolutos e percentuais. Constatou-se grande satisfação dos pacientes quanto a humanização do atendimento, segundo a avaliação das dimensões dignidade (100\%), comunicação (100\%), aspectos interpessoais (100\%), empatia (100\%), receptividade (100\%) e cuidado (95\%). A compreensão da perspectiva dos pacientes acerca do atendimento fisioterapêutico, proporcionará conhecimento e respaldo aos fisioterapeutas a respeito da sua conduta, e possibilitará o desenvolvimento de um ambiente humanizado.

Palavras-chave: Assistência hospitalar; Especialidade de fisioterapia; Humanização da assistência; Unidades de Terapia Intensiva.

Abstract

The importance of humanized physiotherapeutic care in the Intensive Care Unit is widely known, as well as the perceptions of professionals and family members about humanization. However, the perspective of the patient, who is actually being assisted, is still little known. Based on this, this study aims to describe the perspective of hospitalized patients about physical therapy care in the Intensive Care Unit, considering aspects of humanization. This is a crosssectional, descriptive study, with a quantitative approach. A questionnaire was used consisting of two blocks of closed questions, the first for the knowledge of sociodemographic data and the second for knowledge of the patient's
\end{abstract}


perspective about physical therapy care. The population was composed of 20 adult patients, aged 18 years or older, admitted to the ICU for at least 24 hours, lucid and oriented, and assisted by physiotherapy. A simple descriptive analysis was carried out and the results were presented as absolute numbers and percentages. It was verified that the patients were very satisfied with the humanization of care, according to the evaluation of the dimensions dignity $(100 \%)$, communication $(100 \%)$, interpersonal aspects $(100 \%)$, empathy (100\%), receptivity $(100 \%)$ and care $(95 \%)$. Understanding the patients' perspective about physical therapy care will provide knowledge and support to physical therapists regarding their conduct and will enable the development of a humanized environment.

Keywords: Hospital care; Physical therapy specialty; Humanization of assistance; Intensive Care Units.

\section{Resumen}

La importancia de la atención fisioterapéutica humanizada en la Unidad de Cuidados Intensivos es ampliamente conocida, así como las percepciones de los profesionales y familiares sobre la humanización. Sin embargo, la perspectiva del paciente, que es quien realmente recibe la asistencia, sigue siendo poco conocida. Con base en ello, este estudio tiene como objetivo describir la perspectiva de los pacientes hospitalizados sobre la atención fisioterapéutica en la Unidad de Cuidados Intensivos, considerando aspectos de humanización. Se trata de un estudio transversal y descriptivo, con un enfoque cuantitativo. Se utilizó un cuestionario compuesto por dos bloques de preguntas cerradas, el primero para el conocimiento de los datos sociodemográficos y el segundo para el conocimiento de la perspectiva del paciente sobre la atención de fisioterapia. La población estaba formada por 20 pacientes adultos, de 18 años o más, ingresados en la UCI durante al menos 24 horas, lúcidos y orientados, y asistidos por fisioterapia. Se realizó un análisis descriptivo simple y los resultados se presentaron como números absolutos y porcentajes. Los pacientes se mostraron muy satisfechos con la humanización de la atención, según la valoración de las dimensiones dignidad (100\%), comunicación $(100 \%)$, aspectos interpersonales $(100 \%)$, empatía $(100 \%)$, receptividad $(100 \%)$ y atención $(95 \%)$. Comprender la perspectiva de los pacientes sobre la atención de fisioterapia proporcionará conocimientos y apoyo a los fisioterapeutas en relación con su conducta, y permitirá el desarrollo de un entorno humanizado.

Palabras clave: Atención hospitalaria; Especialidad de fisioterapia; Humanización de la atención; Unidades de Cuidados Intensivos.

\section{Introdução}

A Unidade de Terapia Intensiva (UTI) é um ambiente destinado a pacientes graves ou de risco, que necessitam de assistência contínua e vigilante, sendo admitidos com diferentes perfis clínicos (Marques et al., 2020). O atendimento prestado ao paciente nesse âmbito está sempre em discussão e constante evolução, objetivando executar sempre as melhores condutas e oferecer o suporte mais adequado e eficaz. Para isso, além das diversas tecnologias presentes, a UTI conta também com uma equipe multiprofissional, na qual o fisioterapeuta está inserido (Brasil, 2017; Ceregato et al., 2021).

O profissional da Fisioterapia tem como objetivo proporcionar independência funcional ao paciente. Dessa forma, atua na reabilitação e prevenção de distúrbios dos sistemas osteomioarticular, cardiovascular e neurológico (Feliciano et al., 2012; Rotta et al., 2018; Lima et al., 2020), além de favorecer o bom funcionamento do sistema respiratório, fazendo uso da Ventilação Mecânica quando necessário (Bini et al., 2018).

No entanto, a assistência fisioterapêutica na terapia intensiva não deve estar fundamentada apenas no cuidado fisiopatológico, tendo em vista que os pacientes apresentam também problemas psicossociais como angústia, ansiedade e medo, gerados pela permanência na UTI (Nogueira et al., 2017). A partir disso, fica clara a necessidade da humanização, definida como a capacidade de tratar e se relacionar com respeito, atenção e cuidado, com um olhar além da patologia, mais amplo e empático. $\mathrm{O}$ atendimento fisioterapêutico deve ser capaz de reduzir dificuldades encontradas pelos pacientes durante o período de internação na UTI, através de um relacionamento mais humano e condutas mais cuidadosas (Machado \& Soares, 2016).

Apesar da discussão acerca da humanização ser cada vez mais frequente na literatura (Almeida Neto et al., 2012; Luiz et al., 2017; Sanches et al., 2016), a perspectiva do paciente em relação ao atendimento humanizado ainda é pouco conhecida. Diante disso, esse estudo objetiva descrever a perspectiva dos pacientes internados acerca da assistência fisioterapêutica na Unidade de Terapia Intensiva, considerando aspectos da humanização. 


\section{Metodologia}

Este estudo faz parte de um projeto maior intitulado: "O Cuidado Fisioterapêutico e a Humanização nas Unidades de Terapia Intensiva". O projeto foi examinado e aprovado pelo Comitê de Ética e Pesquisa da Universidade Estadual do Sudoeste da Bahia (UESB) sob parecer 3.050.221 e CAAE 99476218.5.0000.0055. Trata-se de um estudo de corte transversal, com pacientes internados nas três UTIs gerais e adultas de um hospital público do interior da Bahia. Cada uma das três UTIs conta com 10 leitos destinados a pacientes adultos, clínicos ou cirúrgicos, de diversas patologias.

A amostra foi definida por conveniência e constituída por 20 participantes voluntários e maiores de 18 anos, determinados a partir dos critérios de inclusão: estar internado em uma das UTIs do referido hospital, por período maior ou igual a 24 horas; estar lúcido e orientado, com capacidade de verbalização oral e/ou escrita preservadas, de acordo com a Escala de Coma de Glasgow (Brennan et al., 2018) e o Mini Exame do Estado Mental (MEEM) (Bertolucci et al., 1994); ter recebido 3 atendimentos fisioterapêuticos ou mais durante o período de internação; concordar em participar deste estudo.

Foi assegurado aos participantes a total confidencialidade dos seus dados, impossibilitando sua identificação. Os mesmos também foram informados do seu direito de interromper sua participação na pesquisa a qualquer momento, garantido pela assinatura do Termo de Consentimento Livre e Esclarecido (TCLE), conforme os aspectos éticos apresentados na resolução 466/12 do Conselho Nacional de Saúde (Brasil, 2012).

Os pesquisadores receberam informações diárias sobre pacientes internados que estavam aptos a participar desta pesquisa, sendo a coleta de dados realizada através de uma entrevista, sem a presença de qualquer outro profissional de saúde, para evitar constrangimentos e obrigatoriedades. O questionário utilizado foi elaborado por Lopes e Brito (2009) e adaptado para essa pesquisa, com questões fechadas constituídas por dois blocos (Lopes \& Brito, 2009).

O primeiro bloco se refere ao conhecimento dos dados sociodemográficos dos participantes. Os dados analisados foram: sexo; faixa etária (classificada em 20 a 59 anos e maior ou igual 60 anos); escolaridade (definida baixa até ensino fundamental incompleto, média até ensino médio incompleto e alta a partir do ensino médio completo); estado civil (solteiro, casado e viúvo); tempo de internação (menor ou igual a 7 dias e maior ou igual a 8 dias); atendimentos fisioterapêticos ( 3 a 5 atendimentos e 6 ou mais atendimentos); diagnóstico primário.

O segundo bloco consiste em questões acerca de diferentes dimensões do atendimento humanizado. As dimensões analisadas foram: dignidade (ter um atendimento digno e atencioso); comunicação (receber informações claras e objetivas acerca do seu tratamento); aspectos interpessoais (ser tratado de forma respeitosa e cortês); empatia (ter um atendimento individualizado e empático); receptividade (agilidade do fisioterapeuta em responder às necessidades do paciente); cuidado (condutas fisioterapêuticas realizadas com cautela e atenção). As dimensões foram avaliadas como positiva (humanizada) ou negativa (desumanizada), a partir da perspectiva dos pacientes internados.

Os dados coletados foram organizados, tabulados e analisados através do Software Statistical Package for Social Sciences® (SPSS), versão 22.0 para Windows. Foi realizada uma análise estatística descritiva simples, apresentando informações sobre a frequência dos dados em forma de números absolutos e percentuais.

\section{Resultados}

Na Tabela 1, são apresentadas as características sociodemográficas dos participantes. Notou-se que a maior parte dos entrevistados eram do sexo feminino (55\%), na faixa etária maior ou igual a 60 anos (70\%), com baixo nível de escolaridade (45\%) e casados (40\%). O tempo de internação na Unidade de Terapia Intensiva foi, em sua maioria, menor ou igual a 7 dias $(65 \%)$ e os participantes tinham realizado 6 ou mais atendimentos fisioterapêuticos $(50 \%)$. 
Tabela 1 - Características da população estudada.

\begin{tabular}{|c|c|c|}
\hline & Frequência & $\%$ \\
\hline \multicolumn{3}{|l|}{ Sexo } \\
\hline Feminino & 11 & $55 \%$ \\
\hline Masculino & 09 & $45 \%$ \\
\hline \multicolumn{3}{|l|}{ Faixa Etária (anos) } \\
\hline $20-59$ & 06 & $30 \%$ \\
\hline$\geq 60$ & 14 & $70 \%$ \\
\hline \multicolumn{3}{|l|}{ Escolaridade } \\
\hline Ensino fundamental incompleto & 09 & $45 \%$ \\
\hline Ensino médio incompleto & 04 & $20 \%$ \\
\hline Ensino médio completo & 03 & $15 \%$ \\
\hline Não Respondido & 04 & $20 \%$ \\
\hline \multicolumn{3}{|l|}{ Estado Civil } \\
\hline Solteiro & 07 & $35 \%$ \\
\hline Casado & 08 & $40 \%$ \\
\hline Viúvo & 03 & $15 \%$ \\
\hline Não Respondido & 02 & $10 \%$ \\
\hline \multicolumn{3}{|l|}{ Tempo de Internação (dias) } \\
\hline$\leq 7$ & 13 & $65 \%$ \\
\hline$\geq 8$ & 07 & $35 \%$ \\
\hline \multicolumn{3}{|l|}{ Atendimentos Fisioterapêuticos } \\
\hline 3 a 5 & 08 & $40 \%$ \\
\hline 6 ou mais & 10 & $50 \%$ \\
\hline Não Respondido & 02 & $10 \%$ \\
\hline
\end{tabular}

Fonte: Dados da pesquisa.

A pesquisa foi realizada em UTIs de característica geral, por esse motivo os diagnósticos primários de internação são variados (Figura 1), sendo os diagnósticos neurológicos (30\%) os mais comuns.

Figura 1 - Diagnóstico primário.

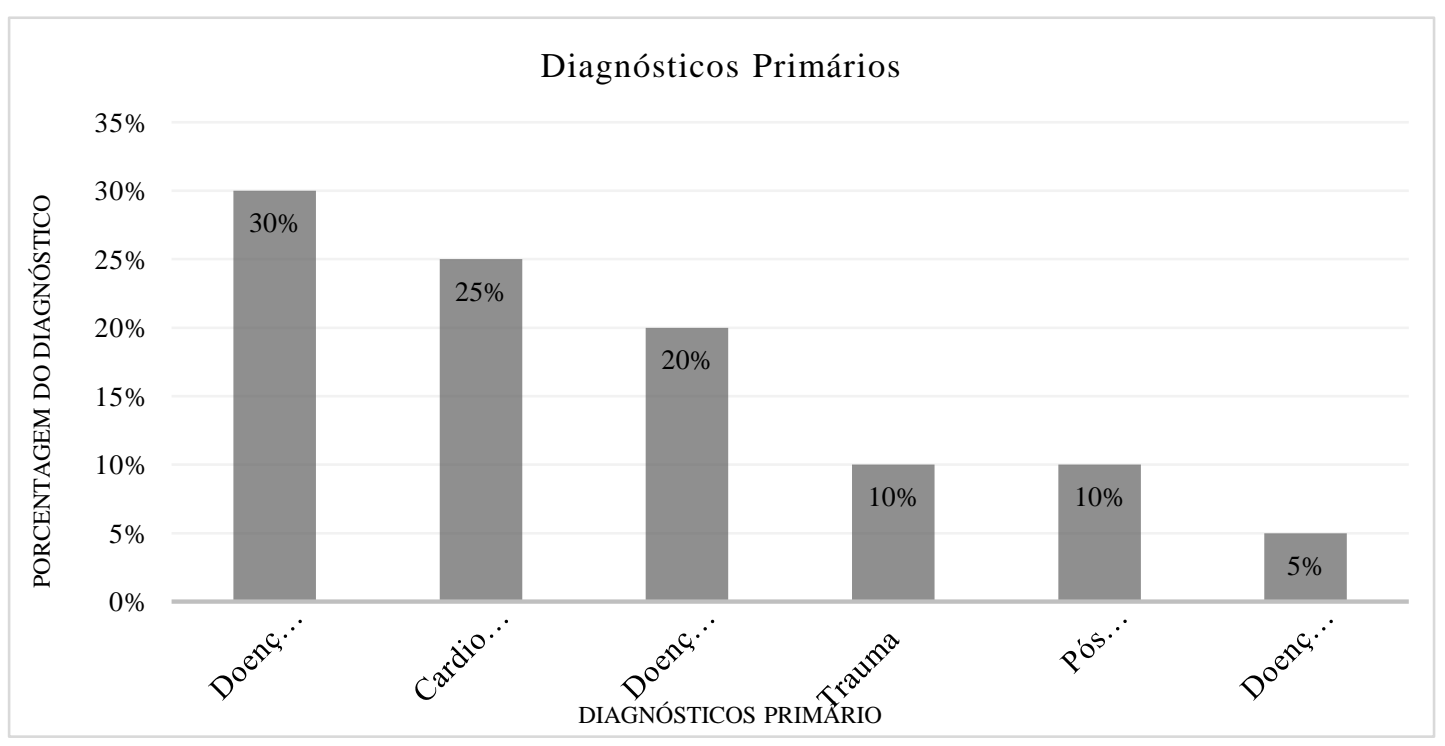

Fonte: Dados da pesquisa. 
Verificou-se que, em relação às dimensões do atendimento fisioterapêutico humanizado, quatro das cinco variáveis foram unânimes no que diz respeito a satisfação dos participantes (Tabela 2), e houve apenas uma avaliação negativa para a dimensão do cuidado (5\%).

Tabela 2 - Perspectiva dos participantes acerca das dimensões do atendimento humanizado.

\begin{tabular}{lcc}
\hline & Frequência & $\%$ \\
\hline Dignidade & 20 & $100 \%$ \\
$\quad$ Positiva & 0 & $0 \%$ \\
Negativa & & \\
Comunicação & 20 & $100 \%$ \\
$\quad$ Positiva & 0 & $0 \%$ \\
$\quad$ Negativa & & \\
Aspectos Interpessoais & 20 & $100 \%$ \\
$\quad$ Positivo & 0 & $0 \%$ \\
$\quad$ Negativa & & $100 \%$ \\
Empatia & 20 & $0 \%$ \\
$\quad$ Positiva & 0 & \\
$\quad$ Negativa & & $100 \%$ \\
Receptividade & 20 & $0 \%$ \\
$\quad$ Positiva & 0 & \\
$\quad$ Negativa & & $95 \%$ \\
Cuidado & 19 & $5 \%$ \\
$\quad$ Positivo & 1 & \\
Negativo & & \\
\hline
\end{tabular}

Fonte: Dados da pesquisa.

A partir dos dados coletados, foi possível verificar que os participantes da pesquisa apresentaram alta taxa de aprovação quanto a assistência fisioterapêutica prestada na UTI, comprovando a prática humanizada nesse ambiente.

\section{Discussão}

A população desse estudo foi composta por 20 participantes internados em Unidades de Terapia Intensiva, predominantemente do sexo feminino, casados e acima dos 60 anos. As principais causas de internação observadas foram as patologias neurológicas e cardiovasculares, com um período de internação de 7 dias ou menos. A maioria dos pacientes realizou 6 ou mais atendimentos fisioterapêuticos, fator que tem importância significativa na redução do tempo de internação e ventilação mecânica, bem como reduz o risco de óbitos por disfunções respiratórias e, consequentemente, os custos hospitalares (Rotta et al., 2018).

Os participantes dessa pesquisa eram principalmente pacientes com baixa escolaridade (45\%), e apesar desse aspecto pressupor um nível inferior de conhecimento dos seus direitos quanto a um atendimento digno (Lopes \& Brito, 2009), também exige do profissional um cuidado e sensibilidade maior quanto às informações que serão passadas aos pacientes, de forma que estas sejam devidamente claras e objetivas e não restem dúvidas acerca da necessidade e forma de execução da assistência dedicada a eles.

A comunicação, dimensão que analisa se os devidos esclarecimentos acerca do seu tratamento foram adequados, foi avaliada como humanizada por $100 \%$ dos participantes. Pesquisas apontam que a comunicação é um fator determinante no que 
diz respeito ao atendimento humanizado. Familiares e profissionais de saúde relataram a importância de ser bem informado e observaram como a falta de comunicação e informações vagas geram insegurança e desconfiança, além de causar desconforto para os pacientes e familiares (Reis et al., 2016; Sanches et al., 2016; Souza et al., 2020).

Outro aspecto igualmente importante da humanização é a dignidade do atendimento recebido. Esse aspecto está pautado no respeito, cuidado e atenção que o paciente recebe durante o seu tratamento, dessa forma, considera como o paciente se sentiu ao ser tratado pelo profissional. $\mathrm{O}$ atendimento digno engloba também aspectos interpessoais, ou seja, a forma com que o fisioterapeuta se refere ao paciente, demonstrando interesse, ânimo, e respeito ao falar com o mesmo (Lopes \& Brito, 2009; Luiz et al., 2017).

Os participantes desta pesquisa, de forma unânime, relataram que o atendimento prestado a eles na UTI foi digno, assim como avaliaram os aspectos interpessoais como 100\% humanizado. Em discussões acerca do que é a humanização, a dignidade e o respeito estão presentes em muitos dos relatos dos profissionais de saúde, constatando o conhecimento teórico dos profissionais acerca dessas dimensões do atendimento humanizado (Luiz et al., 2017; Machado \& Soares, 2016). Verificou-se a partir do presente estudo que esse conhecimento é devidamente aplicado na prática clínica e com expressiva satisfação dos pacientes.

A humanização também está sempre associada a empatia, definida como a capacidade de se colocar no lugar do outro, de entender o paciente como uma pessoa, com suas diferentes características e contextos, e não tratar apenas a patologia que ele apresenta (Machado \& Soares, 2016; Rodrigues et al., 2019). Lopes e Brito (2009) observaram em sua pesquisa que na dimensão da empatia houve $88,6 \%$ de respostas positivas dos participantes, apresentando alto grau de satisfação dos pacientes quanto a habilidade empática dos profissionais que os atenderam. Semelhantemente, um alto grau de satisfação dos participantes foi observado nesta pesquisa, porém ainda mais elevado (100\%), comprovando a eficácia e sensibilidade da assistência fisioterapêutica nessa dimensão.

A disposição para ajudar e atender às necessidades dos pacientes, traduzida aqui como receptividade, foi também avaliada por Mondadori et al. (2016) em seu estudo, e obteve unanimidade dos participantes que avaliaram essa característica do atendimento humanizado como $100 \%$ positiva, concordando com o presente estudo que apresentou a mesma taxa de satisfação dos pacientes quanto a receptividade. Apesar de menos citado, esse é um fator que pesa quanto a análise do atendimento humanizado, e tem base na Política Nacional de Humanização (PNH) que garante que os profissionais devem ter uma conduta de acolhimento integral ao paciente (Brasil, 2013).

Um estudo descritivo observacional realizado em 2019 obteve relatos dos profissionais de saúde acerca da sua visão do atendimento humanizado, e constataram que o acolhimento integral é uma visão já formada nos profissionais, a partir das alegações de que o atendimento não se resume ao medicar e executar condutas, mas se estende desde os cuidados íntimos até o ouvir atentamente as solicitações e questionamentos dos pacientes (Rodrigues et al., 2019; Ceregato et al., 2021).

No que diz respeito às condutas fisioterapêuticas realizadas na UTI, algumas são conhecidas por seu caráter de desconforto para o paciente, como a terapia de higiene brônquica. Apesar dessas condutas serem muitas vezes necessárias e benéficas para a situação clínica do paciente, o incômodo infligido deve ser considerado no momento da decisão do procedimento a ser realizado (Lopes \& Brito, 2009).

O cuidado ao realizar condutas fisioterapêuticas na UTI foi avaliado neste estudo e teve uma taxa de aprovação de $95 \%$ dos pacientes, resultado que está em concordância com outros estudos da literatura que obtiveram taxas semelhantes de 95\% (Lopes \& Brito, 2009) e 100\% de aprovação (Mondadori et al., 2016), constatando a humanização no cuidado ao realizar as condutas necessárias.

O conhecimento teórico acerca de questões gerais da humanização foi avaliado em um estudo descritivo, realizado com diversos profissionais de saúde que atuam no ambiente da terapia intensiva e com um intervalo de 5 anos. A partir dos resultados, 
constatou-se uma mudança positiva no que diz respeito ao conhecimento dos profissionais da Fisioterapia acerca desse domínio (Cangussu et al., 2020). Através do presente estudo, foi possível verificar que a humanização está cada vez mais frequente na rotina dos fisioterapeutas dentro da Unidade de Terapia Intensiva e pode ser constatada através das perspectivas dos pacientes assistidos.

\section{Conclusão}

Conforme os resultados obtidos através dessa pesquisa, foi possível observar que a assistência fisioterapêutica no ambiente da Terapia Intensiva foi considerada humanizada pelos pacientes internados, tendo em vista suas excelentes avaliações quanto a dignidade do atendimento, a clareza expressa na comunicação do profissional da fisioterapia, os aspectos interpessoais da assistência, a empatia demonstrada durante o período de internação, a receptividade do fisioterapeuta com os pacientes e o cuidado com que as condutas foram realizadas.

O estudo apresentou como aspecto limitante a entrevista realizada para coleta de dados, que pode ter deixado os participantes receosos quanto a suas respostas, principalmente por ainda estarem recebendo assistência na UTI, entretanto, durante a entrevista nenhum profissional da equipe estava presente e os participantes foram assegurados de que seus dados não seriam divulgados.

O presente estudo traz à discussão a perspectiva do paciente acerca do atendimento fisioterapêutico que lhe é concedido dentro da Unidade de Terapia Intensiva, e possibilita que eles expressem sua satisfação e insatisfação em relação a aspectos humanizantes da assistência, ao mesmo tempo que os leva a conhecer seus direitos quanto a um atendimento humanizado.

Apesar de poucas avaliações negativas observadas nesse estudo, faz-se necessária a realização de novas pesquisas na área, com populações maiores e que abranjam outras áreas geográficas. A partir da perspectiva dos pacientes acerca do atendimento, será possível proporcionar conhecimento e respaldo aos fisioterapeutas a respeito da sua conduta, e possibilitar o desenvolvimento de um ambiente mais humanizado.

\section{Referências}

Almeida Neto, A. B. D., Evangelista, D. T. O., Tsuda, F. C., Piccinin, M. D. J., Roquejani, A. C., \& Kosour, C. (2012). Percepção dos familiares de pacientes internados em Unidade de Terapia Intensiva em relação à atuação da Fisioterapia e à identificação de suas necessidades. Fisioterapia e Pesquisa, 19(4), 332338. https://doi.org/10.1590/s1809-29502012000400007

Bertolucci, P. H., Brucki, S. M., Campacci, S. R., \& Juliano, Y. (1994). O Mini-Exame do Estado Mental em uma população geral: impacto da escolaridade. Arquivos de Neuro-Psiquiatria, 52(1), 01-07. https://doi.org/10.1590/s0004-282x1994000100001

Bini, R., Pletsch, A. H. M., Reis, A. B. O. D., Souza, F. D., \& Cuiabano, T. K. (2018). Perfil dos Idosos Atendidos pela Fisioterapia na UTI Geral do Hospital Geral Universitário - HGU de Cuiabá/MT. Journal of Health Sciences, 20(1), 25. https://doi.org/10.17921/2447-8938.2018v20n1p25-28

Brasil, Ministério da Saúde. (2012). Dispõe sobre diretrizes e normas regulamentadoras de pesquisas envolvendo seres humanos (Resolução $\mathrm{n}^{\circ}$ 466, de 12 de dezembro de 2012). https://www.inca.gov.br/sites/ufu.sti.inca.local/files//media/document//resolucao-cns-466-12.pdf

Brasil, Ministério da Saúde. (2013). Política Nacional de Humanização. https://bvsms.saude.gov.br/bvs/publicacoes/politica_nac ional_humanizacao_pnh_folheto.pdf

Brasil, Ministério da Saúde. (2017). Institui o cuidado progressivo ao paciente crítico ou grave com os critérios de elegibilidade para admissão e alta, de classificação e de habilitação de leitos de Terapia Intensiva Adulto, Pediátrico, Unidade Coronariana, Queimados e Cuidados Intermediários Adulto e Pediátrico no âmbito do Sistema Único de Saúde (SUS) (Portaria $\mathrm{n}^{\circ} \quad 895$, de $31 \quad$ de março de 2017). http://bvsms.saude.gov.br/bvs/saudelegis/gm/2017/prt0895_26_04_2017.html

Brennan, P. M., Murray, G. D., \& Teasdale, G. M. (2018). Simplifying the use of prognostic information in traumatic brain injury. Part 1: The GCS-Pupils score: an extended index of clinical severity. Journal of Neurosurgery, 128(6), 1612-1620. https://doi.org/10.3171/2017.12.jns172780

Cangussu, D. D. D., Santos, J. F. D. S., \& Ferreira, M. D. C. (2020). Humanização em unidade de terapia intensiva na percepção dos profissionais da saúde. Revista de Divulgação Científica Sena Aires, 167-174. https://doi.org/10.36239/revisa.v9.n2.p167a174

Ceregato, A. C. L., Ribeiro, E. R., Souza, J. M. D., \& Aquim, E. E. (2021). Perfil de competências de fisioterapeutas atuantes em unidades de terapia intensiva. Revista Sustinere, 9. https://doi.org/10.12957/sustinere.2021.47080 
Research, Society and Development, v. 10, n. 9, e21310917892, 2021

(CC BY 4.0) | ISSN 2525-3409 | DOI: http://dx.doi.org/10.33448/rsd-v10i9.17892

Feliciano, V. A., Albuquerque, C. G., Andrade, F. M. D., Dantas, C. M., Lopez, A., Ramos, F. F., Silva, P. F. D. S., \& França, E. E. T. (2012). A influência da mobilização precoce no tempo de internamento na Unidade de Terapia Intensiva. ASSOBRAFIR Ciência, 3(2), 31-42. https://www.cpcrjournal.org/journal/assobrafir/article/5de125150e8825d94d4ce1d8

Lima, A. M. S. D., Brandão, D. C., Barros, C. E. S. R., Richtrmoc, M. K. D. F., Andrade, A. D. F. D. D., \& Campos, S. L. (2020). Knowledge of physiotherapists working in adult ICU on contraindications to mobilization. Fisioterapia Em Movimento, 33. https://doi.org/10.1590/1980-5918.033.ao72

Lopes, F. M., \& Brito, E. S. (2009). Humanização da assistência de fisioterapia: estudo com pacientes no período pós-internação em unidade de terapia intensiva. Revista Brasileira de Terapia Intensiva, 21(3), 283-291. https://doi.org/10.1590/s0103-507x2009000300008

Luiz, F. F., Caregnato, R. C. A., \& Costa, M. R. D. (2017). Humanization in the Intensive Care: perception of family and healthcare professionals. Revista Brasileira de Enfermagem, 70(5), 1040-1047. https://doi.org/10.1590/0034-7167-2016-0281

Machado, E. R., \& Soares, N. V. (2016). Humanização em UTI: Sentidos e Significados Sob a Ótica da Equipe de Saúde. Revista de Enfermagem Do CentroOeste Mineiro, 6(3). https://doi.org/10.19175/recom.v6i3.1011

Marques, C. R. G., Santos, M. R., Passos, K. S., Naziazeno, S. D. S., Sá, L. A., \& Santos, E. S. (2020). Caracterização do perfil clínico e sociodemográfico de pacientes admitidos em uma Unidade de Terapia Intensiva. Interfaces Científicas - Saúde e Ambiente, 8(2). https://doi.org/10.17564/2316-3798.2020v8n2

Mondadori, A. G., Zeni, E. D. M., Oliveira, A. D., Silva, C. C. D., Wolf, V. L. W., \& Taglietti, M. (2016). Humanização da fisioterapia em Unidade de Terapia Intensiva Adulto: estudo transversal. Fisioterapia e Pesquisa, 23(3), 294-300. https://doi.org/10.1590/1809-2950/16003123032016

Nogueira, J. J. D. Q., Ferreira, J. D. A., Albuquerque, A. M. D., \& Agra, G. (2017). Fatores agravantes e atenuantes à percepção de morte em UTI: a visão dos pacientes Aggravating and mitigating factors to death perception in the ICU: a vision of patients. Revista de Pesquisa Cuidado é Fundamental Online, 9(1), 5156. https://doi.org/10.9789/2175-5361.2017.v9i1.51-56

Reis, L. C. C., Gabarra, L. M., \& Moré, C. L. O. O. (2016). As repercussões do processo de internação em UTI adulto na perspectiva de familiares. Temas Em Psicologia, 24(3), 815-828. https://doi.org/10.9788/tp2016.3-03

Rodrigues, Y. S., Santana, R. P., \& Pinheiro, C. P. O. (2019). Humanização na Unidade de Terapia Intensiva Adulta. CORPVS, 1(1), 5-20.

Rotta, B. P., Silva, J. M. D., Fu, C., Goulardins, J. B., Pires-Neto, R. D. C., \& Tanaka, C. (2018). Relationship between availability of physiotherapy services and ICU costs. Jornal Brasileiro de Pneumologia, 44(3), 184-189. https://doi.org/10.1590/s1806-37562017000000196

Sanches, R. D. C. N., Gerhardt, P. C., Rêgo, A. D. S., Carreira, L., Pupulim, J. S. L., \& Radovanovic, C. A. T. (2016). Perceptions of health professionals about humanization in intensive care unit adult. Escola Anna Nery - Revista de Enfermagem, 20(1). https://doi.org/10.5935/1414-8145.20160007

Souza, D. B., Silva, R. M., Ferreira, J. V. P., Soria, V. L., Trindade, M. L., \& Flores, C. M. L. (2020). Perspectiva de familiares acerca da Atenção Multiprofissional em Unidade de Terapia Intensiva. Saúde (Santa Maria), 46(2). https://doi.org/10.5902/2236583441733 